\title{
Gene-gene interaction network analysis of ovarian cancer using TCGA data
}

\author{
Huanchun Ying ${ }^{1 *}$, Jing Lv², Tianshu Ying ${ }^{1}$, Shanshan Jin ${ }^{1}$, Jingru Shao', Lili Wang ${ }^{1}$, Hongying X ${ }^{3}$, Bin Yuan² \\ and Qing Yang ${ }^{1}$
}

\begin{abstract}
Background: The Cancer Genome Atlas (TCGA) Data portal provides a platform for researchers to search, download, and analysis data generated by TCGA. The objective of this study was to explore the molecular mechanism of ovarian cancer pathogenesis.
\end{abstract}

Methods: Microarray data of ovarian cancer were downloaded from TCGA database, and Limma package in R language was used to identify the differentially expressed genes (DEGs) between ovarian cancer and normal samples, followed by the function and pathway annotations of the DEGs. Next, NetBox software was used to for the gene-gene interaction (GGl) network construction and the corresponding modules identification, and functions of genes in the modules were screened using DAVID.

Results: Our studies identified 332 DEGs, including 146 up-regulated genes which mainly involved in the cell cycle related functions and cell cycle pathway, and 186 down-regulated genes which were enriched in extracellular region par function, and Ether lipid metabolism pathway. GGI network was constructed by 127 DEGs and their significantly interacted 209 genes (LINKERs). In the top 10 nodes ranked by degrees in the network, 5 were LINKERs. Totally, 7 functional modules in the network were selected, and they were enriched in different functions and pathways, such as mitosis process, DNA replication and DNA double-strand synthesis, lipid synthesis processes and metabolic pathways. AR, BRCA1, TFDP1, FOXM1, CDK2, and DBF4 were identified as the transcript factors of the 7 modules.

Conclusion: our data provides a comprehensive bioinformatics analysis of genes, functions, and pathways which may be involved in the pathogenesis of ovarian cancer.

Keywords: Differentially expressed genes, Function and pathway annotation, Gene-gene interaction network, Functional modules

\section{Introduction}

Ovarian cancer remains a significant public health burden, with the highest mortality rate of all the gynecological cancer, accounting for about three percent of all cancers in women [1]. Despite advances in surgery and chemotherapy, ovarian cancer in the majority of women will return and become resistant to further treatments [2]. Thus, identifying variations of differentially expressed genes (DEGs) will allow for the possibility of administering alternate therapies that may improve outcomes.

\footnotetext{
* Correspondence: huanchunyiing@hotmail.com

'Department of Gynecology and Obstetrics, Shengjing Hospital of China Medical University, No.36, Sanhao Street, Heping District, Shenyang, Liaoning Province 110004, China

Full list of author information is available at the end of the article
}

Bioinformatics analysis provides a first large scale integrative view of the aberrations in high grade serous ovarian cancer, with surprisingly simple mutational spectrum [3]. Previous studies have examined the role of genetic variation associated with the susceptibility, progression, treatment response, and survival of ovarian cancer $[4,5]$. It has been shown that high grade ovarian cancer is characterized by TP53 mutations in almost all tumors [6]. KRAS-variant is found to be a genetic marker for increased risk of developing ovarian cancer [7]. Genes related with cell cycle, lipid metabolism and cytoskeletal structure are screened as the treatment targets for ovarian cancer [8]. 
TCGA (The Cancer Genome Atlas) is a national collaborative program where different tumor types are being collected, and each tumor is being characterized using a variety of genome-wide platforms [9]. TCGA has recently complemented its first formal analysis of the genomic and clinical data from the ovarian carcinoma project.

In this study, we downloaded the microarray data of ovarian cancer form TCGA database for the identification of DEGs, and the annotation of abnormal functions and pathways in ovarian cancer. A gene-gene interaction (GGI) network was constructed using NetBox software, comprised by DEGs and their significant interacted genes. The network was further studied for its functional modules.

\section{Methods}

\section{Gene expression profiles}

We downloaded gene expression data batch8_9 from TCGA project webpage (https://tcga-data.nci.nih.gov/ tcga/), including 38 ovarian cancer samples and 8 matched normal samples. Data levels are assigned for data type, platform and center in TCGA. The data we downloaded consisted of level 1-4, and we chose level 3 (for Segmented or Interpreted Data) for further study. Median method was used for the standardizations of the original data.

\section{Screening of DEGs}

We applied the Limma package in $\mathrm{R}$ language, a linear regression model, to select the DEGs in ovarian cancer samples compared with the normal samples [10]. Only the genes with $\mathrm{p}$-value $<0.05$ and $\mid \log$ Fold Chance (FC) $\mid>1.5$ were screened out as DEGs.

\section{Functions and pathways enrichment of DEGs}

The significant functions and pathways of DEGs was assessed based on the GO (Gene Ontology) [11] and KEGG (Kyoto Encyclopedia of Genes and Genomes) [12] annotations using Gestalt (Gene Set Analysis Toolkit) software. False discovery rate (FDR) less than 0.05 was set as the cut-off criteria.

\section{GGI network construction}

The interactions between DEGs were searched using NetBox software. NetBox is a toolkit used in the establishment of interaction network based on public database of HPRD (Human Protein Reference Database) [13], Reactome [14], NCI-Nature Pathway Interaction database (PID) [15], as well as the MSKCC Cancer Cell Map [16]. Firstly, we obtained a global network. Then, DEGs were mapped onto the network, as well as the genes significantly interacted with these DEGs. Finally, we established the GGI network using the DEGs and their significant interaction nodes according to the assigned criteria ( $\mathrm{p}<0.05$ and shortest path threshold was 2). We also calculate the degrees of the nodes by igraph package in $\mathrm{R}$ language, and identified the key nodes with high degrees.

\section{Functional modules analysis}

Beside the GGI network under the assigned criteria, NetBox also divided the network into modules. Next, DAVID (Database for Annotation, Visualization and Integration Discovery) was used to identify the overrepresented GO categories and KEGG pathways of the modules with FDR less than 0.05. DAVID could provide a high-throughput and integrated data-mining environment, and analysis gene lists derived from high-throughput genomic experiments.

\section{Results}

\section{DEGs screening}

The expressions profiles of the whole 46 samples included 12042 genes. By limma package, a total of 332 genes were selected as the DEGs between ovarian and normal samples, of which 146 were up-regulated.

\section{Significant functions and pathways of DEGs}

The enriched functions and pathways of up-regulated DEGs are listed in Table 1. Functions that related to cell cycle, nucleotide binding, and mitosis of up-regulated DEGs were enriched, while cell cycle pathway was enriched. GO: 0044421 (extracellular region part) and hsa00565: Ether lipid metabolism pathway was the only enriched function and pathway of down-regulated DEGs, respectively.

\section{GGI network}

A global network including 9264 genes and 68111 lines were obtained after the searching by NetBox. After screening, the GGI network was established (Figure 1), comprising of the selected DEGs and their interaction nodes (named as LINKER). There were 946 nodes in the network, including 209 LINKERs, 127 DEGs and 4105 lines. We calculated the degrees of the nodes in the network, and the top 10 nodes are listed in Table 2. LINKERs such as AHCTF1, B9D2, CASC5, and CCDC99 were found to be closely related with DEGs in the databases $(\mathrm{p}<0.05)$.

\section{Functional modules mining and analysis}

Totally, 32 functional modules in the PPI network were given by the NetBox software, 7 of which having more than 10 genes (Figure 2). The results of functions and pathways clustering of the 7 modules are displayed in Table 3 . Genes in module 1 were mainly participated in the mitosis process occurred in nucleus. Module 2 genes were mainly involved in DNA replication and DNA 
Table 1 Significant GO terms and KEGG pathways of up-regulated DEGs

\begin{tabular}{|c|c|c|c|c|}
\hline Category & Term & Count & $P$ value & FDR \\
\hline GOTERM_BP_FAT & GO:0007049 cell cycle & 49 & $1.60 \mathrm{E}-28$ & $2.58 \mathrm{E}-25$ \\
\hline GOTERM_BP_FAT & GO:0000278 mitotic cell cycle & 37 & $8.84 \mathrm{E}-28$ & $1.42 \mathrm{E}-24$ \\
\hline GOTERM_BP_FAT & GO:0022403 cell cycle phase & 38 & 3.07E-27 & 4.94E-24 \\
\hline GOTERM_BP_FAT & GO:0000279 M phase & 35 & 4.47E-27 & 7.20E-24 \\
\hline GOTERM_BP_FAT & GO:0007067 mitosis & 29 & $8.44 \mathrm{E}-25$ & 1.36E-21 \\
\hline GOTERM_BP_FAT & GO:0000280 nuclear division & 29 & $8.44 \mathrm{E}-25$ & $1.36 \mathrm{E}-21$ \\
\hline GOTERM_BP_FAT & GO:0000087 M phase of mitotic cell cycle & 29 & $1.41 \mathrm{E}-24$ & $2.26 \mathrm{E}-21$ \\
\hline GOTERM_BP_FAT & GO:0022402 cell cycle process & 40 & $1.42 \mathrm{E}-24$ & 2.29E-21 \\
\hline GOTERM_BP_FAT & GO:0048285 organelle fission & 29 & $2.62 \mathrm{E}-24$ & 4.22E-21 \\
\hline GOTERM_BP_FAT & GO:0051301 cell division & 30 & $1.92 \mathrm{E}-22$ & 3.09E-19 \\
\hline GOTERM_MF_FAT & GO:0005524 ATP binding & 29 & 7.57E-06 & 0.0101086 \\
\hline GOTERM_MF_FAT & GO:0032559 adenyl ribonucleotide binding & 29 & $9.78 \mathrm{E}-06$ & 0.0130621 \\
\hline GOTERM_MF_FAT & GO:0030554 adenyl nucleotide binding & 29 & $2.59 \mathrm{E}-05$ & 0.0345973 \\
\hline GOTERM_MF_FAT & GO:0001883 purine nucleoside binding & 29 & $3.42 \mathrm{E}-05$ & 0.0456417 \\
\hline GOTERM_CC_FAT & GO:0015630 microtubule cytoskeleton & 30 & $1.73 \mathrm{E}-15$ & $2.21 \mathrm{E}-12$ \\
\hline GOTERM_CC_FAT & GO:0005819 spindle & 17 & $1.31 \mathrm{E}-13$ & 1.63E-10 \\
\hline GOTERM_CC_FAT & GO:0044430 cytoskeletal part & 31 & $3.05 \mathrm{E}-10$ & $3.80 \mathrm{E}-07$ \\
\hline GOTERM_CC_FAT & GO:0005694 chromosome & 22 & $3.52 \mathrm{E}-10$ & 4.38E-07 \\
\hline GOTERM_CC_FAT & GO:0043228 non-membrane-bounded organelle & 52 & $9.48 \mathrm{E}-10$ & $1.18 \mathrm{E}-06$ \\
\hline GOTERM_CC_FAT & GO:0043232 intracellular non-membrane-bounded organelle & 52 & $9.48 \mathrm{E}-10$ & $1.18 \mathrm{E}-06$ \\
\hline GOTERM_CC_FAT & GO:0044427 chromosomal part & 19 & 5.36E-09 & $6.66 \mathrm{E}-06$ \\
\hline GOTERM_CC_FAT & GO:0005856 cytoskeleton & 33 & $1.27 \mathrm{E}-07$ & $1.58 \mathrm{E}-04$ \\
\hline GOTERM_CC_FAT & GO:0000793 condensed chromosome & 11 & $1.68 \mathrm{E}-07$ & 2.09E-04 \\
\hline GOTERM_CC_FAT & GO:0000777 condensed chromosome kinetochore & 8 & $6.43 \mathrm{E}-07$ & 7.99E-04 \\
\hline KEGG_PATHWAY & hsa04110:Cell cycle & 17 & $5.33 \mathrm{E}-15$ & $5.46 \mathrm{E}-12$ \\
\hline
\end{tabular}

GO: Gene Ontology; KEGG: Kyoto Encyclopedia of Genes and Genomes; DEGs: differentially expressed genes; BP: biological process; MF: molecular function; CC: cellular component.

double-strand synthesis, affecting the enzymatic activity participated. Functions that are related with lipid synthesis processes and formation of intracellular membranes were enriched in module 3. Four cancer related pathways were enriched in module 4, and genes in this module can affect cell cycle by influencing the transcriptional function. Genes in module 6 were associated with wound healing process outside the cells. In addition, genes in module 7 were associated with some metabolic pathways, influencing the activities of proteolytic enzyme and ligase. No functions and pathways were found to be related with genes in module 5 .

Besides module 7, the other 6 modules all had the down-regulated genes, such as CETN2 and CDKN1A. These genes might be suppressed in the tumorgenesis of ovarian cancer, and the biological functions and processes of them might be alternated by other promoted genes.

In all, $6 \mathrm{TFs}$ were identified in the 7 modules using TRANSFAC: FOXM1 in module 1; CDK2 and DBF4 in module 2; AR, BRCA1, and TFDP1 in module 4. We got another 36 adjusted cancer-related TFs by searching TRED database.

\section{Discussion}

Ovarian is the most lethal of all reproductive system cancers and presents a real challenge. In order to explore the molecular mechanism of ovarian cancer pathogenesis, we downloaded the gene expression profiles of ovarian cancer in the TCGA database. In the selected 332 DEGs, 146 were up-regulated genes and 186 were down-regulated genes. Cell cycle related functions, such as mitotic cell cycle and $\mathrm{M}$ phase; nucleotide binding related functions, such as adenyl ribonucleotide binding and purine nucleoside binding; mitosis related functions, included spindle and chromosome, and cell cycle pathway was enriched of up-regulated DEGs. In accordance with the study of Wan et al. that the up-regulated genes of ovarian carcinogenesis are related to cell cycling 


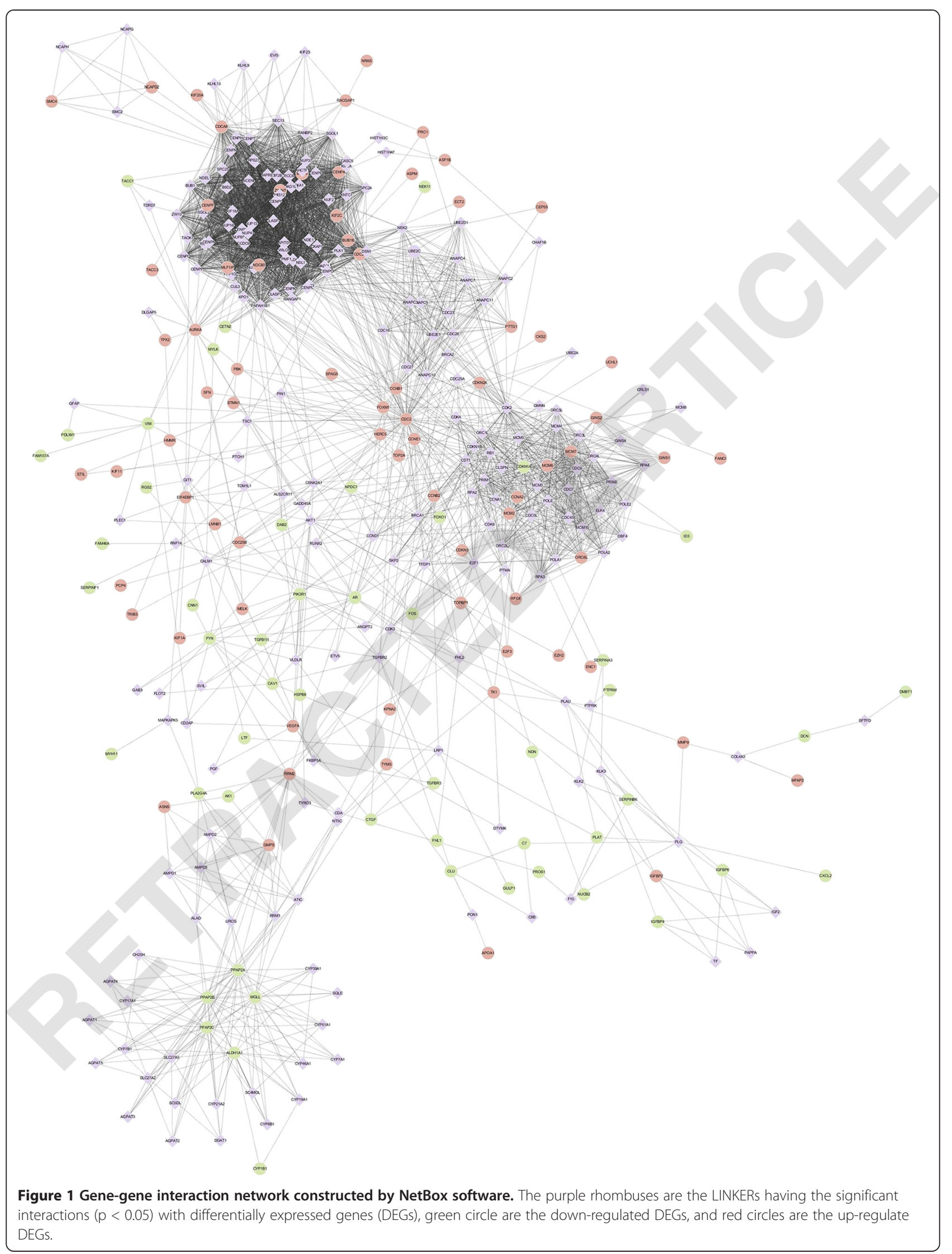


Table 2 Top 10 nodes in the gene-gene interaction network

\begin{tabular}{llll}
\hline Rank & Node & Degree & Mark \\
\hline 1 & CDK2 & 93 & ALTERED \\
2 & AHCTF1 & 91 & LINKER \\
3 & APITD1 & 90 & ALTERED \\
4 & B9D2 & 90 & LINKER \\
5 & CASC5 & 89 & LINKER \\
6 & CCDC99 & 89 & LINKER \\
7 & CENPC1 & 85 & ALTERED \\
8 & CENPE & 83 & ALTERED \\
9 & CENPH & 80 & ALTERED \\
10 & CENPI & 80 & LINKER \\
\hline
\end{tabular}

Note: nodes marked as LINKER are the genes having significant interactions $(p<0.05)$ with differentially expressed genes (DEGs), and nodes marked with ALTERED are the DEGs.

functions [17]. GO: 0044421 (extracellular region part) and hsa00565: Ether lipid metabolism pathway was the only enriched function pathway of down-regulated DEGs, respectively. Thus, we could infer that it is the changes of the related functions and pathways that caused the tumorgenesis of the ovarian cancer.

There were 209 LINKERs out of 336 nodes in the GGI network, as well as 5 LINKER genes in the top10 genes with high degrees in the network. LINKERs are the genes which were not identified to be abnormally expressed in ovarian cancer, but significantly interacted with the DEGs. LINKERs, such as AHCTF1, B9D2, CASC5, and CCDC99 were screened based on the literatures recorded in HPRD, Reactome, PID Interaction and MSKCC Cancer Cell Map, and they were genes in module 1 . These LINKERs participated indirectly in the tumorgenesis and development of ovarian cancer by the interaction with DEGs. The abundance of LINKERs in the GGI network proved the complications of interactions between genes in ovarian cancer. AHCTF1, also known as Elys/AKNA, is originally identified as a putative transcription factor involved in mouse haematopoiesis [18]. AHCTF1 is up-regulated in ductal breast carcinomas [19], and is reported to be a risk factor for cervical cancer [20]. Mutations in B9D2 (B9 protein domain 2) has been linked to human genetic diseases [21]. CASC5 (cancer sensitibity candidate 5) has been shown to be expressed in many human cancer cell lines and in several primary human tumors [22], and CASC5 Note MLL gene and D40 gene are reported to be translocated each other in three cases of leukemia [23]. CCDC99 (coiled-coil domain containing 99) is predicted to be a mitotic spindle protein, and is over-expressed in lung cancer tumor tissues [24].

Genes such as CETN2 and CDKN1A were the downregulated genes screened in the functional modules of the

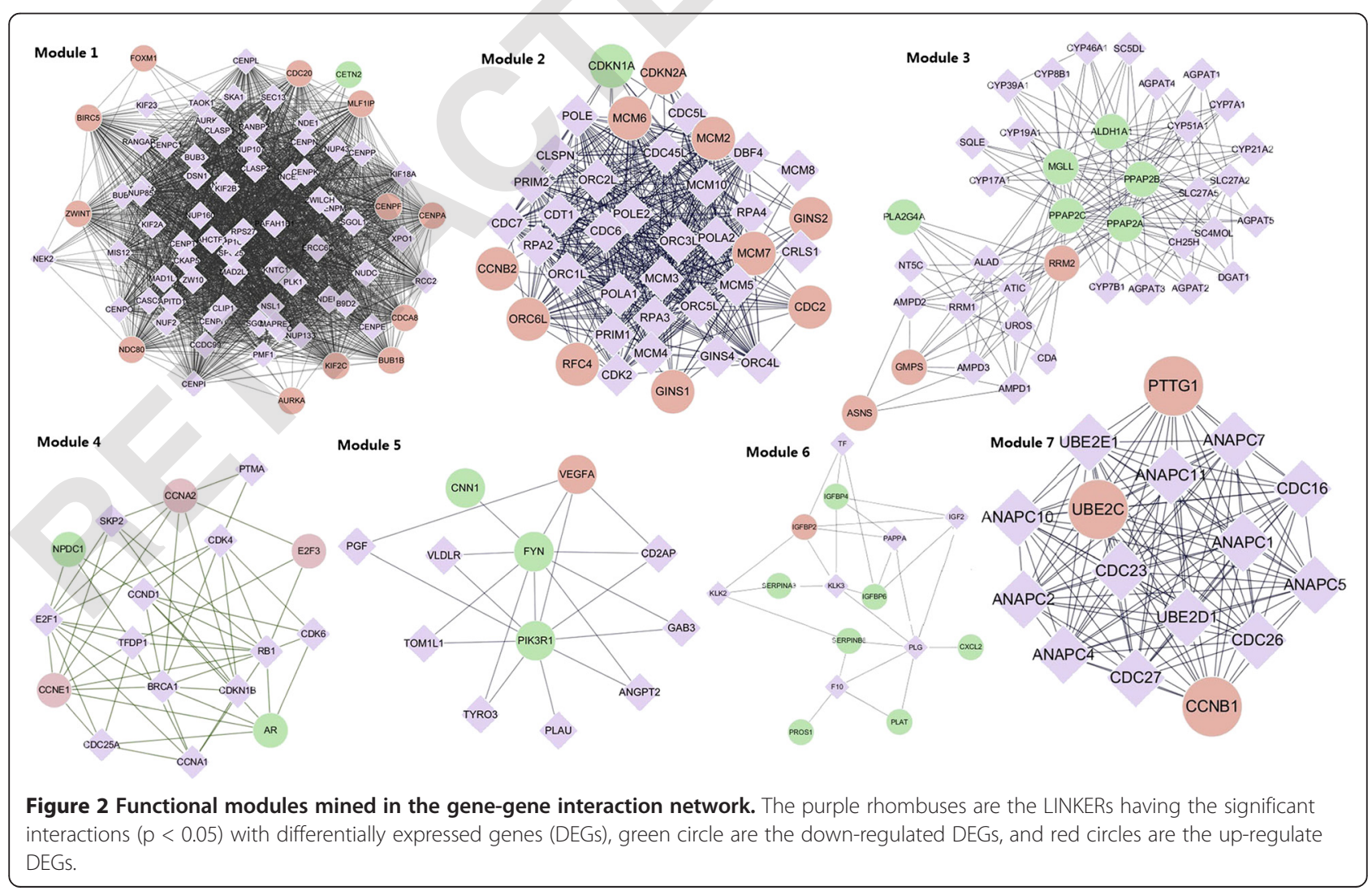


Table 3 Significant GO terms and KEGG pathways of the 7 functional modules

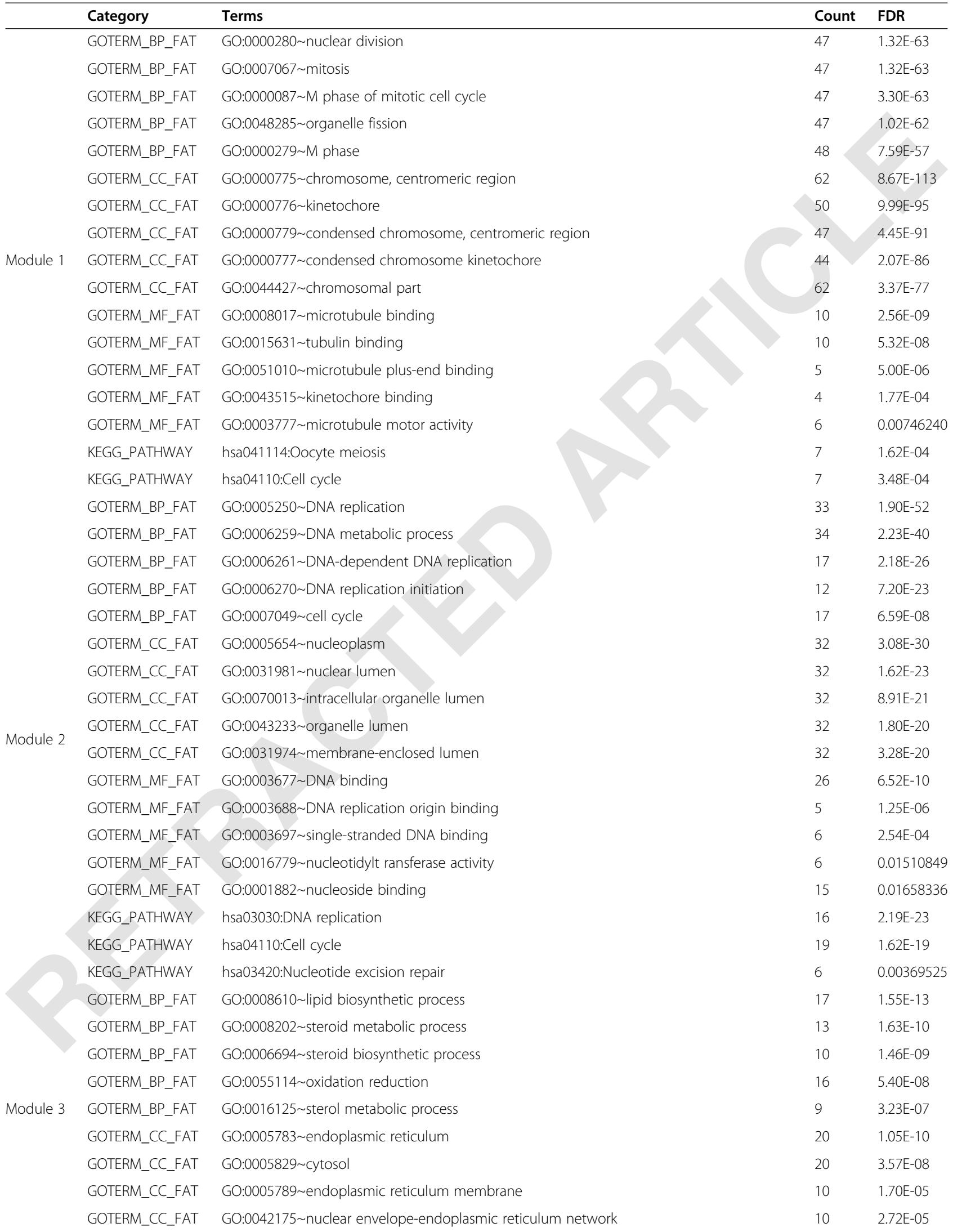


Table 3 Significant GO terms and KEGG pathways of the 7 functional modules (Continued)

\begin{tabular}{|c|c|c|c|c|}
\hline & GOTERM_CC_FAT & GO:0005792 microsome & 9 & $1.12 \mathrm{E}-04$ \\
\hline & GOTERM_MF_FAT & GO:0008395 steroid hydroxylase activity & 8 & 7.37E-12 \\
\hline & GOTERM_MF_FAT & GO:0005506 iron ion binding & 13 & 4.58E-08 \\
\hline & GOTERM_MF_FAT & GO:0046027 phospholipid:diacylglycerol acyltransferase activity & 5 & $3.56 \mathrm{E}-07$ \\
\hline & GOTERM_MF_FAT & GO:001641 1 acylglycerol O-acyltransferase activity & 6 & $5.50 \mathrm{E}-07$ \\
\hline & GOTERM_MF_FAT & GO:0020037 heme binding & 9 & 2.00E-06 \\
\hline & KEGG_PATHWAY & hsa00561:Glycerolipid metabolism & 9 & $5.32 \mathrm{E}-07$ \\
\hline & KEGG_PATHWAY & hsa00120:Primary bile acid biosynthesis & 7 & $6.29 \mathrm{E}-07$ \\
\hline & KEGG_PATHWAY & hsa00565:Ether lipid metabolism & 8 & $2.94 \mathrm{E}-06$ \\
\hline & KEGG_PATHWAY & hsa00564:Glycerophospholipid metabolism & 8 & 3.56E-04 \\
\hline & GOTERM_BP_FAT & GO:0007049 cell cycle & 14 & $8.67 \mathrm{E}-12$ \\
\hline & GOTERM_BP_FAT & GO:0051329 interphase of mitotic cell cycle & 9 & 7.36E-11 \\
\hline & GOTERM_BP_FAT & GO:0051325 interphase & 9 & $9.31 \mathrm{E}-11$ \\
\hline & GOTERM_BP_FAT & GO:0000278 mitotic cell cycle & 11 & $7.71 \mathrm{E}-10$ \\
\hline & GOTERM_BP_FAT & GO:0022402 cell cycle process & 12 & $1.00 \mathrm{E}-09$ \\
\hline & GOTERM_CC_FAT & GO:0005654 nucleoplasm & 10 & 4.75E-05 \\
\hline & GOTERM_CC_FAT & GO:0031981 nuclear lumen & 10 & 0.00340842 \\
\hline & GOTERM_CC_FAT & GO:0070013 intracellular organelle lumen & 10 & 0.01893931 \\
\hline IV & GOTERM_CC_FAT & GO:0043233 organelle lumen & 10 & 0.02288144 \\
\hline & GOTERM_CC_FAT & GO:0031974 membrane-enclosed lumen & 10 & 0.02691056 \\
\hline & GOTERM_MF_FAT & GO:0016563 transcription activator ac & 7 & $3.96 \mathrm{E}-04$ \\
\hline & KEGG_PATHWAY & hsa04110:Cell cycle & 13 & $2.04 \mathrm{E}-15$ \\
\hline & KEGG_PATHWAY & hsa05222:Small cell lung canc & 9 & 8.99E-09 \\
\hline & KEGG_PATHWAY & hsa05200:Pathways in cancer & 11 & 7.11E-07 \\
\hline & KEGG_PATHWAY & hsa05220:Chronic myeloid leukemia & 7 & $1.89 \mathrm{E}-05$ \\
\hline & KEGG_PATHWAY & hsa05215:Prostate cancer & 7 & 5.34E-05 \\
\hline & GOTERM_BP_FAT & GO:0009511 response to wounding & 9 & $7.88 \mathrm{E}-06$ \\
\hline & GOTERM_BP_FAT & GO:0042060 wound healing & 5 & 0.03445470 \\
\hline & GOTERM_CC_FAT & GO:0005576 extracellular region & 13 & 2.43E-06 \\
\hline & GOTERM_CC_FAT & GO:0005615 extracellular space & 8 & 0.00156546 \\
\hline & GOTERM_CC_FAT & GO:0044421 extracellular region part & 8 & 0.01488625 \\
\hline & GOTERM_MF_FAT & GO:0005520 insulin-like growth factor binding & 4 & 0.00208348 \\
\hline & GOTERM_MF_FAT & GO:0004252 serine-type endopeptidase activity & 5 & 0.01598712 \\
\hline & GOTERM_MF_FAT & GO:0008236 serine-type peptidase activity & 5 & 0.02826126 \\
\hline & GOTERM_MF_FAT & GO:0017171 serine hydrolase activity & 5 & 0.02952737 \\
\hline & KEGG_PATHWAY & hsa:04610:Complement and coagulation cascades & 4 & 0.03363616 \\
\hline & GOTERM_BP_FAT & $\begin{array}{l}\text { GO:0031145 anaphase-promoting complex-dependent proteasomal ubiquitin-dependent } \\
\text { protein catabolic process }\end{array}$ & 15 & $1.37 \mathrm{E}-29$ \\
\hline & GOTERM_BP_FAT & GO:0051437 positive regulation of ubiquitin-protein ligase activity during mitotic cell cycle & 15 & 2.77E-29 \\
\hline & GOTERM_BP_FAT & GO:0051443 positive regulation of ubiquitin-protein ligase activity & 15 & 4.34E-29 \\
\hline Module 7 & GOTERM_BP_FAT & GO:0051439 regulation of ubiquitin-protein ligase activity during mitotic cell cycle & 15 & $5.41 \mathrm{E}-29$ \\
\hline & GOTERM_BP_FAT & GO:0051351 positive regulation of ligase activity & 15 & $8.31 \mathrm{E}-29$ \\
\hline & GOTERM_CC_FAT & GO:0005654 nucleoplasm & 15 & $5.11 \mathrm{E}-13$ \\
\hline & GOTERM_CC_FAT & GO:0005829 cytosol & 16 & $1.27 \mathrm{E}-12$ \\
\hline & GOTERM_CC_FAT & GO:0031981 nuclear lumen & 15 & $5.65 \mathrm{E}-10$ \\
\hline
\end{tabular}


Table 3 Significant GO terms and KEGG pathways of the 7 functional modules (Continued)

\begin{tabular}{llcc} 
GOTERM_CC_FAT & GO:0005680 anaphase-promoting complex & 6 & $1.60 \mathrm{E}-09$ \\
GOTERM_CC_FAT & GO:0000151 ubiquitin ligase complex & 8 & $3.17 \mathrm{E}-09$ \\
GOTERM_MF_FAT & GO:0004842 ubiquitin-protein ligase activity & 9 & $1.70 \mathrm{E}-12$ \\
GOTERM_MF_FAT & GO:0019787 small conjugating protein ligase activity & 9 & $4.54 \mathrm{E}-12$ \\
GOTERM_MF_FAT & GO:0016881 acid-amino acid ligase activity & 9 & $2.17 \mathrm{E}-11$ \\
GOTERM_MF_FAT & GO:0016879 ligase activity, forming carbon-nitrogen bonds & 9 & $6.71 \mathrm{E}-11$ \\
KEGG_PATHWAY & hsa04120:Ubiquitin mediated proteolysis & 14 & $9.66 \mathrm{E}-17$ \\
KEGG_PATHWAY & hsa04114:Oocyte meiosis & 13 & $1.07 \mathrm{E}-15$ \\
KEGG_PATHWAY & hsa04110:Cell cycle & 13 & $5.29 \mathrm{E}-15$ \\
KEGG_PATHWAY & hsa04914:Progesterone-mediated oocyte maturation & 12 & $9.39 \mathrm{E}-15$ \\
\hline
\end{tabular}

GO: Gene Ontology; KEGG: Kyoto Encyclopedia of Genes and Genomes; FDR: false discovery rate; BP: biological process; MF: molecular function; CC: cellular component.

GGI network. CETN2 is an X-linked gene, it is reported that the down-regulated gene of CETN2 may have tumor suppressive functions in bladder cancer [25]. Somatic alterations of CDKN1A involved in the G1 phase of the cell cycle, are the common events in neoplastic development for multiple tumor types [26]. Thus, the biological processes and pathways of these genes might be alternated by other genes in the development of ovarian cancers.

FOXM1, CDK2, DBF4, AR, BRCA1, and TFDP1 were the TFs identified in the functional modules in GGI network. FOXM1 (Forkhead box M1) is overexpressed in a majority of human tumors, and represents as an attractive therapeutic target in the fight against cancer [27]. Forkhead TFs are intimately involved in the regulation of organismal development, cell differentiation and proliferation, and the interference with FoxM1 activity is believed to contribute to the increase in mitotic errors seen in human diseases such as cancer [28]. CDK2 (cyclin-dependent kinase 2) is found to be overexpressed in ovarian tumors, and is concur with cyclin $\mathrm{E}$ to ovarian tumor development $[29,30]$. DBF4 was a TF in module 2 , and DNA replication was a significant function of module 2, which is consistent with the discovery that DBF4 is involved in the initiation of DNA replication and overexpressed in human cancer cell lines and in many primary tumors compared with the matched normal tissues [31]. The relationship between the TFs AR, BRCA1 and cancer have already been confirmed in the previous studies [32-34]. TFDP1 suppresses the colorectal cancer development by reducing telomerase activity and inhibiting the apoptosis of cells [35].

In conclusion, our data provides a comprehensive bioinformatics analysis of genes and pathways which may be involved in the pathogenesis of ovarian cancer. We have found a total of 332 DEGs, and constructed the GGI interaction networks by these DEGs and their significantly interacted genes. Furthermore, we conducted functional modules analysis in the network.
However, further analyses are still required to unravel their mechanism in the process of tumor genesis and development in ovarian cancer.

\section{Abbreviations}

DEGs: Differentially expressed genes; TCGA: The cancer genome atlas; GGI: Gene-gene interaction; GO: Gene ontology; KEGG: Kyoto encyclopedia of genes and genomes; FDR: False discovery rate; HPRD: Human protein reference database; PID: Pathway Interaction database; DAVID: Database for annotation, visualization and integration discovery; CCDC99: Coiled-coil domain containing 99; FOXM1: Forkhead box M1; CDK2: Cyclin-dependent kinase 2.

\section{Competing interests}

The authors declare that they have no competing interests.

\section{Authors' contributions}

$\mathrm{HY}$ and HX carried out the molecular genetic studies, participated in the sequence alignment and drafted the manuscript. $J$ carried out the immunoassays. TY participated in the sequence alignment with LW. SJ articipated in the design of the study and performed the statistical analysis. JS, BY and QY conceived of the study, and participated in its design and coordination and helped to draft the manuscript. All authors read and approved the final manuscript.

\section{Acknowledgement}

This study was supported by Liaoning Province Doctor Startup Fund of Natural Science Foundation (20071047); The Scientific Research Project of Higher Education Program of Liaoning Provincial Department of Education (2009A724); Liaoning Science and Technology Project (2010225032) and Natural Science Foundation of China (81372486).

\section{Author details}

'Department of Gynecology and Obstetrics, Shengjing Hospital of China Medical University, No.36, Sanhao Street, Heping District, Shenyang, Liaoning Province 110004, China. ${ }^{2}$ Department of Oncology, The fifth Hospital of Shenyang, Shenyang 110023, China. ${ }^{3}$ Department of Gynecology and Obstetrics, The ninth Hospital of Shenyang, Shenyang 110024, China.

Received: 27 September 2013 Accepted: 14 November 2013 Published: 6 December 2013

\section{References}

1. Braun R, Finney R, Yan C, Chen Q-R, Hu Y, Edmonson M, Meerzaman D, Buetow K: Discovery analysis of TCGA data reveals association between germline genotype and survival in ovarian cancer patients. PLoS One 2013, 8:e55037.

2. Grzankowski KS, Carney M: Quality of life in ovarian cancer. Cancer Control 2011, 18:52-58. 
3. Bell D, Berchuck A, Birrer M, Chien J, Cramer D, Dao F, Dhir R, DiSaia P, Gabra H, Glenn P: Integrated genomic analyses of ovarian carcinoma. 2011.

4. Bolton KL, Ganda C, Berchuck A, Pharaoh PD, Gayther SA: Role of common genetic variants in ovarian cancer susceptibility and outcome: progress to date from the ovarian cancer association consortium (OCAC). J Int Med 2012, 271:366-378.

5. Liang D, Meyer L, Chang DW, Lin J, Pu X, Ye Y, Gu J, Wu X, Lu K: Genetic variants in MicroRNA biosynthesis pathways and binding sites modify ovarian cancer risk, survival, and treatment response. Cancer Res 2010, 70:9765-9776.

6. Cancer Genome Atlas Research Network: Integrated genomic analyses of ovarian carcinoma. Nature 2011, 474:609-615.

7. Ratner E, Lu L, Boeke M, Barnett R, Nallur S, Chin LJ, Pelletier C, Blitzblau R, Tassi R, Paranjape T: A KRAS-variant in ovarian cancer acts as a genetic marker of cancer risk. Cancer Res 2010, 70:6509-6515.

8. Ying $H, L V J$, Ying $T, L i J$, Yang Q: Screening of feature genes of the ovarian cancer epithelia with DNA microarray. J Ovar Res 2013, 6:1-8.

9. Chang H, Fontenay GV, Han J, Cong G, Baehner FL, Gray JW, Spellman PT, Parvin B: Morphometic analysis of TCGA glioblastoma multiforme. BMC Bioinformatics 2011, 12:484

10. Gentleman R, Carey V, Huber W, Irizarry R, Dudoit S: Bioinformatics and computational biology solutions using $R$ and bioconductor: vol. 746718470. Statistics for Biology and Health: Springer New York; 2005.

11. Ashburner M, Ball CA, Blake JA, Botstein D, Butler H, Cherry JM, Davis AP, Dolinski K, Dwight SS, Eppig JT: Gene ontology: tool for the unification of biology. Nat Genet 2000, 25:25-29.

12. Kanehisa M: The KEGG database, Novartis found symp. 2002

13. Prasad TK, Goel R, Kandasamy K, Keerthikumar S, Kumar S, Mathivanan S, Telikicherla D, Raju R, Shafreen B, Venugopal A: Human protein reference database-2009 update. Nucleic Acids Res 2009, 37:D767-D772.

14. Joshi-Tope G, Gillespie M, Vastrik I, D'Eustachio P, Schmidt E, de Bono B, Jassal B, Gopinath G, Wu G, Matthews L: Reactome: a knowledgebase of biological pathways. Nucleic Acids Res 2005, 33:D428-D432.

15. Schaefer CF, Anthony K, Krupa S, Buchoff J, Day M, Hannay T, Buetow KH: PID: the pathway interaction database. Nucleic Acids Res 2009, 37:D674-D679.

16. Somwar R, Erdjument-Bromage $H$, Larsson E, Shum D, Lockwood WW, Yan G, Sander C, Ouerfelli O, Tempst PJ, Djaballah H: Superoxide dismutase 1 (SOD1) is a target for a small molecule identified in a screen for inhibitors of the growth of lung adenocarcinoma cell lines. Proc Natl Acad Sci 2011, 108:16375-16380.

17. Wan S-M, LV F, Guan T: Identification of genes and microRNAs involved in ovarian carcinogenesis. Asian Pac J Cancer Prev 2012, 13:3997-4000.

18. Kimura N, Takizawa M, Okita K, Natori O, Igarashi K, Ueno M, Nakashima K, Nobuhisa I, Taga T: Identification of a novel transcription factor, ELYS, expressed predominantly in mouse foetal haematopoietic tissues. Genes Cells 2002, 7:435-446.

19. Turashvili G, Bouchal J, Baumforth K, Wei W, Dziechciarkova M, Ehrmann J, Klein J, Fridman E, Skarda J, Srovnal J: Novel markers for differentiation of lobular and ductal invasive breast carcinomas by laser microdissection and microarray analysis. BMC Cancer 2007, 7:55

20. Perales G, Burquete-García Al, Dimas J, Bahena-Román M, Bermúdez-Morales $\mathrm{VH}$, Moreno J, Madrid-Marina V: A polymorphism in the AT-hook motif of the transcriptional regulator AKNA is a risk factor for cervical cancer. Biomarkers 2010, 15:470-474.

21. Chea E: Regulation of planar cell polarity and Vangl2 trafficking by Tmem $14 a$ 2012.

22. Ghafouri-Fard S, Modarressi M-H: Expression of cancer-testis genes in brain tumors: implications for cancer immunotherapy. Future Oncol 2012, 8:961-987.

23. Takimoto M: Gene section. 2013, 15:1. http://AtlasGeneticsOncology.org.

24. Pacurari M, Qian Y, Porter D, Wolfarth M, Wan Y, Luo D, Ding M, Castranova V, Guo N: Multi-walled carbon nanotube-induced gene expression in the mouse lung: association with lung pathology. Toxicol Appl Pharmacol 2011, 255:18-31.

25. Tatarano S, Chiyomaru T, Kawakami K, Enokida H, Yoshino H, Hidaka H, Yamasaki T, Kawahara K, Nishiyama K, Seki N: miR-218 on the genomic loss region of chromosome $4 \mathrm{p} 15.31$ functions as a tumor suppressor in bladder cancer. Inter J Oncol 2011, 39:13-21.

26. Gayther SA, Song H, Ramus SJ, Kjaer SK, Whittemore AS, Quaye L, Tyrer J, Shadforth D, Hogdall E, Hogdall C: Tagging single nucleotide polymorphisms in cell cycle control genes and susceptibility to invasive epithelial ovarian cancer. Cancer Res 2007, 67:3027-3035.

27. Bhat UG, Halasi M, Gartel AL: Thiazole antibiotics target FoxM1 and induce apoptosis in human cancer cells. PLoS One 2009, 4:e5592.

28. Laoukili J, Stahl M, Medema RH: FoxM1: at the crossroads of ageing and cancer: biochimica et biophysica acta (BBA)-reviews on. Cancer 2007, 1775:92-102.

29. Marone M, Scambia G, Giannitelli C, Ferrandina G, Masciullo V, Bellacosa A Benedetti-Panici P, Mancuso S: Analysis of cyclin E and CDK2 in ovarian cancer: gene amplification and RNA overexpression. Intern J Cancer 1998, 75:34-39.

30. Bast RC, Hennessy B, Mills GB: The biology of ovarian cancer: new opportunities for translation. Nat Rev Cancer 2009, 9:415-428.

31. Montagnoli A, Moll J, Colotta F: Targeting cell division cycle 7 kinase: a new approach for cancer therapy. Clin Cancer Res 2010, 16:4503-4508.

32. Elattar A, Warburton KG, Mukhopadhyay A, Freer RM, Shaheen F, Cross $P$, Plummer ER, Robson CN, Edmondson RJ: Androgen receptor expression is a biological marker for androgen sensitivity in high grade serous epithelial ovarian cancer. Gynecol Oncol 2012, 124:142-147.

33. Audeh MW, Carmichael J, Penson RT, Friedlander M, Powell B, Bell-McGuinn KM, Scott C, Weitzel JN, Oaknin A, Loman N: Oral poly (ADP-ribose) polymerase inhibitor olaparib in patients with $<\mathrm{i}>\mathrm{BRCA} 1</ \mathrm{i}>$ or $<\mathrm{i}>$ $B R C A 2</ i>$ mutations and recurrent ovarian cancer: a proof-of-concept trial. Lancet 2010, 376:245-251.

34. Zhang S, Royer R, Li S, McLaughlin JR, Rosen B, Risch HA, Fan I, Bradley L, Shaw PA, Narod SA: Frequencies of BRCA1 and BRCA2 mutations among 1,342 unselected patients with invasive ovarian cancer. Gynecol Oncol 2011, 121:353-357.

35. Shin SM, Chung YJ, Oh ST, Jeon HM, Hwang LJ, Namkoong H, Kim HK, Cho GW, Hur SY, Kim TE: HCCR-1-interacting molecule "deleted in polyposis 1" plays a tumor-suppressor role in colon carcinogenesis. Gastroenterology 2006, 130:2074-2086

doi:10.1186/1757-2215-6-88

Cite this article as: Ying et al:: Gene-gene interaction network analysis of ovarian cancer using TCGA data. Journal of Ovarian Research 2013 6:88.

\section{Submit your next manuscript to BioMed Central and take full advantage of:}

- Convenient online submission

- Thorough peer review

- No space constraints or color figure charges

- Immediate publication on acceptance

- Inclusion in PubMed, CAS, Scopus and Google Scholar

- Research which is freely available for redistribution 